\title{
METODOLOGÍAS ACTIVAS EN EL LOGRO DEL DESARROLLO DE COMPETENCIAS EMPRENDEDORAS CON EL APOYO DE LAS TICS
}

\author{
Adrian Jose Barrios Vergara $\mathrm{A}^{\mathbf{1}}$; \\ María Fernanda Dominguez Buelvas $\mathrm{B}^{2}$; \\ Edna Margarita Esteban Regino $C^{3}$;
}

\begin{abstract}
The research was framed within the duty that universities in Colombia have to promote the entrepreneurial culture and entrepreneurship, according to Law 1014 of 2006 of the Republic of Colombia. The objective was to analyze the effectiveness of the implementation of active methodologies and the critical model with a focus on competencies in the achievement of the development of competencies in the students of the seventh semester as entrepreneurs to create companies with the support of ICTs, From the course "Elective I Digital, Social and Solidarity Entrepreneurship", a methodology of participative action research (PAI) was developed in five moments, it was demonstrated that it is possible to promote in students the generation of ideas, strengthen the competences and skills to identify business opportunities, producing proposals of original solutions to various real problems.
\end{abstract}

Keywords: Entrepreneurial skills, digital entrepreneurship, solidarity entrepreneurship, creative thinking, flipped classroom

\section{Resumen}

La investigación se enmarcó en el deber que tienen las universidades en Colombia de fomentar la cultura empresarial y el emprendimiento, según la Ley 1014 de 2006 de la Republica de Colombia, como objetivo se planteó analizar la efectividad de la implementación de metodologías activas y el modelo crítico con enfoque por competencias en el logro del desarrollo de competencias en los estudiantes de VII semestre como emprendedores para crear empresas con el apoyo de las TIC, desde el curso "Electiva I Emprendimiento digital, social y solidario", se desarrolló una metodología de investigación de acción participativa (IAP) en cinco momentos, se demostró que se puede promover en los estudiantes la generación de ideas, potenciar las competencias y habilidades para identificar oportunidades de negocio, produciendo propuestas de soluciones originales a diversos problemas reales.

Palabras-claves: competencias emprendedoras, emprendimiento digital, emprendimiento solidario, pensamiento creativo, aula invertida

\footnotetext{
1 Programa de Ingeniería de Sistemas - Universidad Cooperativa de Colombia -UCC, Colombia, adrian.barriosv@campusucc.edu.co

2 Programa de Ingeniería de Sistemas - Universidad Cooperativa de Colombia -UCC, Colombia, maria.dominguezb@campusucc.edu.co

${ }^{3}$ Programa de Ingeniería de Sistemas - Universidad Cooperativa de Colombia - UCC, Colombia, edna.estebanr@campusucc.edu.co
}

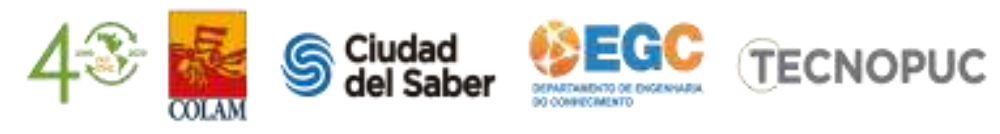




\section{INTRODUCCIÓN}

Ante el reto de fomentar la cultura empresarial, la Universidad Cooperativa de Colombia - UCC emprendió la exigente tarea de ajustarse a las dinámicas contemporáneas, impulsada por un gran deseo de transformación, razón por la cual el equipo investigador desde el programa de ingeniería de sistemas de la Universidad Cooperativa de Colombia UCC - Montería, se propuso analizar la efectividad de la implementación de metodologías activas (Fernández, 2006) y el modelo crítico con enfoque por competencias (Unigarro, 2017) en el logro del desarrollo de competencias en los estudiantes de VII semestre como emprendedores para crear empresas con el apoyo de las TIC, desde el curso "Electiva I Emprendimiento digital, social y solidario", se seleccionaron las metodologías activas por ser consideradas herramientas adecuadas para preparar al ingeniero de hoy a afrontar los retos que la sociedad actual, desde la importancia de enseñarle al estudiante a "saber”, “saber ser y estar" y a "saber hacer”, logrando que los alumnos sean protagonistas de su aprendizaje y el modelo crítico con enfoque por competencias por articular los postulados de la pedagogía critica con un enfoque por competencias.

La investigación se enmarcó en el deber que tienen las universidades en Colombia de fomentar la cultura empresarial y el emprendimiento, según la Ley 1014 de 2006 de la Republica de Colombia (Congreso de Colombia, 2006), además en el ámbito educativo de los países desarrollados, la formación de emprendedores se ha convertido en un asunto de política pública, por lo que se reconoce que la formación universitaria debe fomentar la cultura empresarial, ya que un profesional empresarial accede a mayores posibilidades de articulación a la vida productiva, siendo así, la enseñanza del emprendimiento se reconoce como una oportunidad de formar nuevos líderes que le aporten al desarrollo de sus regiones (Andrade \& Preciado, 2013).

\section{CONTEXTO TEÓRICO}

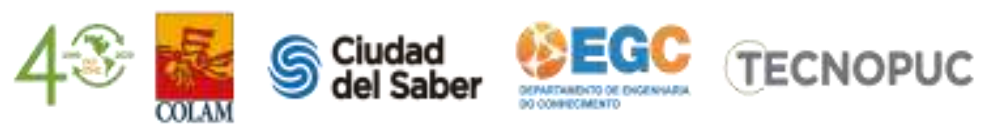


Uno de los retos actuales de la educación superior es como lograr que el estudiante aprenda mejor, sin embargo el proceso enseñanza aprendizaje es considerado la interacción de muchas variables, entre ella las que se relacionan con el docente, el aprendiz y la tarea, por lo que el análisis debe realizarse de manera multidimensional, en cuanto al docente se debe analizar la motivación hacia el proceso de instrucción, en el aprendiz los conocimientos previos, la motivación hacia la tarea y las estrategias de aprendizaje (Saíz \& Montero, 2016), por lo que el presente documento hace una reflexión sobre la efectividad de la implementación de metodologías activas y el modelo crítico con enfoque por competencias en el logro del desarrollo de competencias en los estudiantes de VII semestre como emprendedores para crear empresas con el apoyo de las TIC, para ello se tuvieron en cuenta estrategias didácticas que orientaran al logro del desarrollo de las competencias en los estudiantes como emprendedores para crear su propio negocio, entre ellas se destacan Think-Up (Pensamiento Creativo), la investigación documental y científica, el modelo de aula invertida (Flipped Clasroom) y el modelo crítico con enfoque por competencias.

\section{Metodología activas}

Paños (Paños, 2017) hace una revisión sobre las metodologías que se deben emplear para desarrollar la competencia del emprendimiento, el cual no se refiere solo a la creación de nuevas empresas y/o negocios, sino a la capacidad de resolver problemas en cualquier situación en diversos contextos, lo que requiere un conjunto de habilidades y destrezas que comprenden: creatividad, liderazgo, trabajo en equipo, innovación, toma de decisiones, búsqueda de oportunidades, compromiso, capacidad para trabajar en red, pensamiento estratégico, capacidad de negociación, capacidad de persuasión, orientación al logro, asunción de riesgos, adaptación al entorno, automotivación, iniciativa y visión de futuro. Por consiguiente, son necesarias las metodologías activas para el logro de competencias emprendedoras, donde el alumno adopte un papel activo, participativo y se sitúe en el centro del proceso de enseñanza---aprendizaje, siendo él protagonista y el profesor guía, asesor y/o facilitador, para ello se

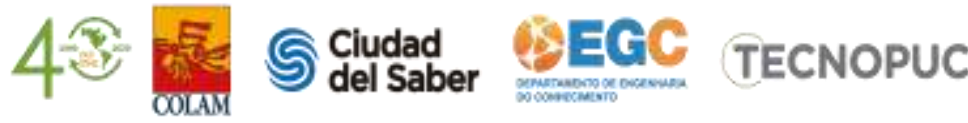


consideran el estudio de casos, hasta el aprendizaje basado en problemas, el aprendizaje basado en proyectos, las simulaciones y juegos y el aprendizaje cooperativo.

\section{Estrategias y técnicas didácticas}

En (UNIMINUTO, 2017)se hace una recopilación de 31 procedimientos didacticos para aportar a las experiencias educativas, entre ellos: Aprendizaje Basado en Problemas (ABP), donde los estudiantes trabajan en grupos pequeños para sintetizar y construir el conocimiento para resolver los problemas, favoreciendo el desarrollo de habilidades para el análisis y síntesis de información, incentivando el trabajo colaborativo. Aprendizaje orientado a proyectos (AOP)), Se involucra a los a estudiantes en proyectos complejos y del mundo real con el fin de obtener un aprendizaje significativo. El pensamiento de diseño (design thinking) utilizada la resolución de problemas de forma práctica y creativa, combinando la empatía, la creatividad y el razonamiento para satisfacer las necesidades de formación de los estudiantes, basada en la inspiración, la ideación y la implementación. Investigación documental, se utiliza la observación y la reflexión sobre realidades o teorías usando diferentes documentos, se indaga, interpreta y se presentan datos e informaciones sobre un tema específico utilizando para ello un método de análisis. Think - Up (pensamiento creativo) promueve conceptos o nuevas asociaciones entre ideas, para producir soluciones originales a diversos problemas reales.

\section{El modelo de aula invertida (Flipped Clasroom)}

Según (Romero, Artal, García, \& Artacho, 2017), es un modelo en el cual el alumnado recibe una parte instruccional de la enseñanza fuera del aula y las horas de clase se dedican a resolver dudas sobre las tareas encomendadas, discutir a fondo sobre aquello que más le cuesta comprender (aprendizaje face-to-face) o trabajar en problemas y proyectos, el modelo fomenta que el alumno sea más autónomo (aprender a aprender) y obtenga un aprendizaje más significativo, en él los estudiantes se ayudan entre sí (aprendizaje cooperativo) y el profesorado establece su rol como guía, llegando incluso a "desaparecer" en determinadas tareas autónomas, donde resulta imprescindible la implementación y el uso de las herramientas TIC, combinando

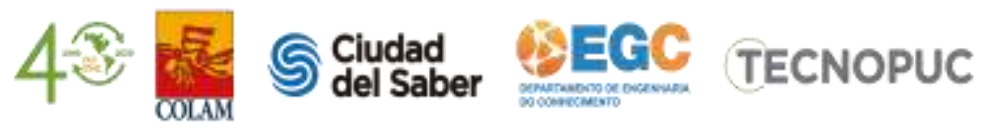


eficientemente recursos virtuales y físicos. Dicho modelo se complementa con el desarrollo de las competencias de expresión oral, comunicación y transmisión de ideas mediante la utilización de dos de las técnicas de presentación más actuales: Elevator Pitch y Pecha Kucha.

\section{Modelo crítico con enfoque por competencias}

Se encuentra enmarcado en la Pedagogía crítica, como estrategia para responder desde la educación a las demandas del mundo, educando para el trabajo y creando espacios para que las personas puedan desarrollarse plenamente sin que deban emplearse para lograrlo (Unigarro, 2017).

\section{Emprendimiento}

El emprendimiento se soporta en la construcción de proyectos de realización personal; representa una herramienta muy útil para que las personas piensen y estructuren el modo de hacer realidad sus metas o propósitos de vida, impulsa a la multiplicación de su riqueza o bienestar de vida y que se ampara en la Ley 1014 de 2006 colombiana, la cual busca fomentar la cultura emprendedora en todos los planteles educativos del país, mediante una formación dirigida a inculcar "una manera de pensar y actuar orientada hacia la creación de riqueza".

\section{Emprendimiento en Colombia}

Actualmente el emprendimiento en Colombia cuenta con cifras alentadoras, entre 144 países ocupa el puesto 66 y ocupa el cuarto puesto en el listado de países con mayor número de emprendimientos innovadores en fase inicial, antecedido por Chile, Dinamarca y Sudáfrica (Revista emprendedores , 2017). Gracias al Ministerio de las Tecnologías de la Información y las Comunicaciones MinTIC, se han implementado programas que fortalecen los componentes del ecosistema digital, lo que da paso a una nueva generación de emprendedores digitales que desarrollan ideas de negocio de base tecnológica (MinTic, 2016), donde su principal programa es Apps.co, el cual se concentra en brindar talleres de entrenamiento para mejorar las habilidades en identificar oportunidades, siguiendo los principios de "Lean Startup", con los que se busca construir proyectos viables a través del uso de tres técnicas: el diseño de modelo de negocios, el desarrollo de clientes y el desarrollo ágil (UniMOOC, 2014). Es así como (Andrade \& Preciado, 2013) aseguran que la enseñanza del

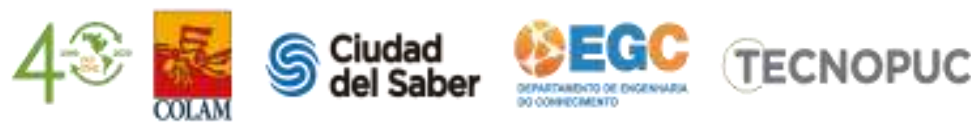


emprendimiento se reconoce como una oportunidad de formar nuevos líderes que le aporten al desarrollo de sus regiones, lo que genera un avance en el rol tradicional del docente frente a su labor académica, consistiendo en crear nuevas propuestas pedagógicas y didácticas ligadas al desarrollo significativo de los aprendizajes.

\section{Emprendimiento Solidario}

El emprendimiento solidario es una alternativa para unir esfuerzos, con miras a que los emprendedores de una misma actividad económica logren consolidarse organizacionalmente para crecer rápidamente y sobrevivir en un mundo globalizado (Rodriguez, 2016).

\section{MATERIALES Y MÉTODOS}

Para la realización de la presente investigación se implementó una metodología de investigación de acción participativa (IAP) (Diccionario de Acción Humanitaria y Cooperación al Desarrollo, 2006) que la población estudiada debía realizar en cinco momentos: Un primer momento se aplicó un método de investigación y aprendizaje colectivo de la realidad, donde los estudiantes debían realizar un análisis crítico de las necesidades sociales de su comunidad dentro de jornadas de ideación o talleres de design thinking (Cuevas, 2018) como estrategia para el pensamiento creativo y la innovación con la participación de líderes sociales y expertos en el desarrollo de emprendimientos sociales y tecnológicos.

Un segundo momento los participantes estructuraron el marco referencias del caso de estudio seleccionado en un primer momento a partir de la búsqueda de información documental (Esteban, 2016). Un tercer momento para enunciar el problema de investigación, describir su estado actual, narrar los hechos o antecedentes que caracterizan dicha situación, mostrar sus implicaciones (Bernal, 2010) y soluciones basadas en el uso de las TIC (elaboración de textos académicos: ensayos y resúmenes) (CREA Tecnologico de Monterrey, 2011). Un cuarto momento donde se formularon preguntas orientadas a dar respuesta al problema de la investigación a partir de la implementación de las metodologías activas (Aprendizaje Basado

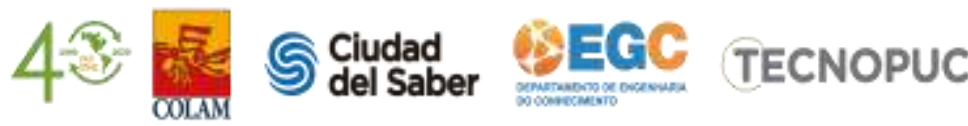


en Problemas (ABP), el Aprendizaje cooperativo - colaborativo y el Aprendizaje orientado a proyectos (AOP)) (Fernández, 2006).

Finalmente para analizar la efectividad de la implementación de metodologías activas y el modelo crítico con enfoque por competencias en el logro del desarrollo de competencias en los estudiantes de VII semestre de ingeniería de sistemas como emprendedores para crear empresas con el apoyo de las TICs, se implementó un quinto momento donde los participantes presentaron sus propuestas de solución en distintos eventos de emprendimientos para que fueran valoradas por expertos, aplicando el modelo de aula invertida (Flipped Clasroom) una metodología propuesta para la enseñanza del emprendimiento, haciendo uso de la técnica del Elevator Pitch (Romero, Artal, García, \& Artacho, 2017), al tiempo que empleaban los principios de la metodología "Lean Startup" (Edinson, Smorsgard, Wang, \& Abrahamsson, 2018).

\section{RESULTADOS}

La implementación sistemática de la metodología descrita durante siete semestres consecutivos: $2017-10,2017-20,2018$-10, 2018 -20, 2019-10, $2019-20,2020-10$ ha permitido formar en emprendimiento digital social y solidario a 93 estudiantes y se han generado 28 ideas de negocios ver tabla 1

Tabla 1 - Total estudiantes e ideas de negocios de emprendimiento digital, social y solidario

\begin{tabular}{ccc}
\hline Semestre & Nro. estudiantes & Nro. Ideas de Negocios \\
\hline $2017-10$ & 13 & 4 \\
\hline $2017-20$ & 13 & 4 \\
\hline $2018-10$ & 15 & 4 \\
\hline $2018-20$ & 10 & 3 \\
\hline $2019-10$ & 19 & 5 \\
\hline $2019-20$ & 9 & 3 \\
\hline $2020-01$ & 14 & 5 \\
\hline Total 7 semestres & 93 & 28 \\
\hline
\end{tabular}


De manera consecutiva las ideas de negocio han representado y han obtenido el primer lugar para el programa de sistemas de sistemas en el concurso Rymel Serrano Uribe de la sede en 2017, 2018 y 2019 con las propuesta TRUEQUEANDO, LOVE YOUR LIFE; NATURIMS y REDRECYCLE, lo que permitió representar a la sede a nivel nacional, donde NATURISM fue seleccionado en segundo lugar a nivel nacional en la categoría de Ideas de Negocio para Organizaciones de Economía Solidaria (INDESCO, 2018).

Adicional a ello las ideas de negocios de emprendimiento digital social y solidario generadas se ha presentado en otros eventos de emprendimiento como: la jornada \#JOC18 XXXIII Jornada de Opinión Cooperativa - InnovAcción en la era digital en Cartagena, 25 al 27 de abril - 2018 (ASCOOP, 2018), Digital Bank de Bogotá (DIGITAL BANK, 2018), Hackathon transformación digital del sector productivo $24-26$ de julio en Montería (Transformación digital del sector productivo, 2018), Techstars Startup Weekend Colombia 2018 Nodo Córdoba - 7-9 de septiembre - 2018 (SiKUANI.NET, 2018), La FET 2018Montería (Camara de Comercio-Montería, 2018).

Es así que con el fortalecimiento de las competencias de los estudiantes como emprendedores para crear empresas con el apoyo de las TICs, se crea el Semillero de Investigación de Emprendimiento Digital, Social y Solidario - SIEDSS, un semillero que nace de las iniciativas de ideas de negocios soportadas desde los principios de la economía solidaria el 2 de febrero del 2018, con la participación de 24 estudiantes del programa de ingeniería de sistemas - Montería, con el objetivo de generar espacios de discusión e investigación en temas concernientes al emprendimiento digital, social y solidario, que permitan fortalecer tanto las competencias investigativas, como también desarrollar habilidades en los estudiantes como emprendedores para crear su propio negocio, articulado al Grupo de investigación INDESCO.

\section{DISCUSIÓN}

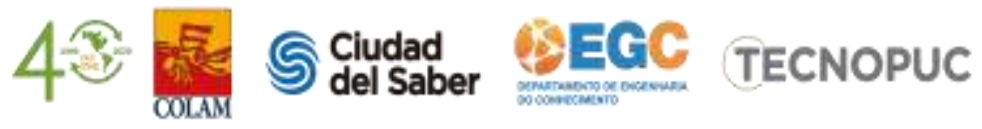


Conforme con lo descrito por (Andrade \& Preciado, 2013), esta investigación coincide en que la formación universitaria debe fomentar la cultura empresarial, puesto que al integrar en el currículo la enseñanza del emprendimiento los estudiantes asumen actitudes de liderazgo para proponer soluciones desde la ingeniería de sistemas y principios de la economía solidaria a las necesidades de su comunidad, lo que le brindó a los estudiantes la posibilidad de articularse a la vida productiva con proyectos que pudieron evaluarse como viables en los diferentes eventos donde participaron. Se pudo evidenciar que, al aplicar propuestas pedagógicas y didácticas ligadas a desarrollo significativo de los aprendizajes, como las metodologías activas como Think-Up (Pensamiento Creativo), la investigación documental y científica propuestas desde UNIMINUTO (2016), el modelo de aula invertida (Flipped Clasroom) aplicada por (Romero, Artal, García, \& Artacho, 2017) y el modelo crítico con enfoque por competencias de (Unigarro, 2017), se pueden desarrollar competencias para detectar nuevas oportunidades para emprender nuevos negocios y desarrollar nuevos productos.

Se pudo comprobar que el emprendimiento en Colombia se encuentra en un buen momento como lo afirma (Revista emprendedores , 2017), y que los estudiantes al proponer soluciones desde la ingeniería de sistemas pueden aprovechar programas que fortalezcan sus emprendimientos digitales como los ofertados por el Ministerio de las Tecnologías de la Información y las Comunicaciones MinTIC (MinTic, 2016), entrenándose en el diseño de modelo de negocios, el desarrollo de clientes y el desarrollo ágil (UniMOOC, 2014), para mejorar las habilidades en identificar oportunidades.

\section{CONCLUSIONES}

Finalmente se alcanzó el objetivo propuesto, al analizar la efectividad de la implementación de metodologías activas y el modelo crítico con enfoque por competencias en el logro del desarrollo de competencias en los estudiantes de VII semestre como emprendedores para crear empresas con el apoyo de las TIC, desde el curso "Electiva I Emprendimiento digital, social y solidario", puesto que los estudiantes presentaron sus propuestas de solución (Pitch) en distintos eventos de emprendimientos para que fueran valoradas por expertos.

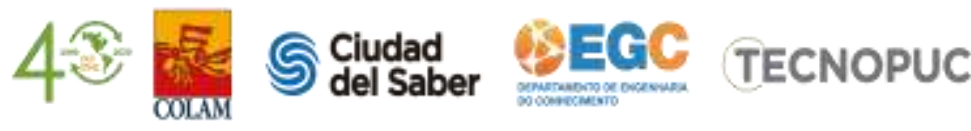


Al combinar diversas estrategias didácticas como: la investigación de acción participativa (IAP), el design thinking, la búsqueda de información documental, la investigación científica, la elaboración de textos académicos, el Aprendizaje Basado en Problemas (ABP), el Aprendizaje cooperativo - colaborativo y el Aprendizaje orientado a proyectos (AOP)), el modelo de aula invertida (Flipped Clasroom), la metodología "Lean Startup”, quedó demostrado que se puede promover en los estudiantes la generación de ideas, potenciar las competencias y habilidades para identificar oportunidades de negocio, produciendo propuestas de soluciones originales a diversos problemas reales, generando en ellos una unidad como expertos investigadores quienes gestaron sus propios proyectos de investigación, transformando su propia realidad y aportando a la construcción de su proyecto de vida.

El principal factor motivador de la estrategia fue el emprendimiento solidario, impulsando a los estudiantes a multiplicar su riqueza o bienestar de vida por medio de la realización de acciones conjuntas, impulsándolos a adoptar la cultura del emprendimiento, desarrollándose como personas con la capacidad de crear, llevar adelante sus ideas, generar bienes y servicios, asumir riesgos y enfrentar problemas e impactar en la sociedad, neutralizando las deficiencias o dificultades que tiene que afrontar el emprendedor individual, optando por la alternativa de unir esfuerzos, con miras a consolidarse organizacionalmente para crecer rápidamente y sobrevivir en un mundo globalizado

Se destaca la competencia que desarrollaron los estudiantes para presentar ideas de negocio asociativo y solidario, proponiendo soluciones a las problemáticas empresariales del sector de la economía solidaria, con propuestas como Truequeando, con la que los estudiantes se proponen diseñar un sistema de información web que le permita a la comunidad universitaria UCC implementar iniciativas de trueque solidario de manera permanente, con el fin de intercambiar bienes y servicios para generar impacto en la comunidad a nivel económico y social, o Naturism, en la que los estudiantes proponen fomentar el turismo, dando a conocer productos y servicios de la comunidad de Caño Grande (APRACAG) ubicada en el municipio de San Bernardo del Viento (Córdoba), la cual se dedica a esta actividad económica de manera

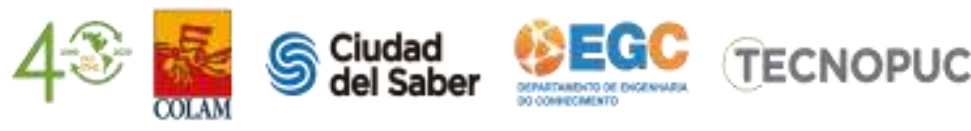


asociativa y solidaria a través de un sistema de información con el fin de que la comunidad pueda percibir mayores ingresos y mejorar su calidad de vida.

\section{REFERENCIAS}

Andrade, M., \& Preciado, W. (2013). PROPUESTA DIDÁCTICA PARA LA ENSEÑANZA DEL EMPRENDIMIENTO. Amazonia Investiga, 32-45.

ASCOOP. (26 de Abril de 2018). \#JOC18 - XXXIII Jornada de Opinión Cooperativa. Obtenido de http://ascoop.coop/joc/img/ConJuevM/index.html

Bernal, C. (2010). Metodologia de la Investigacion. En C. Bernal, Metodologia de la Investigacion (págs. 95-100). Bogota: Pearson.

Camara de Comercio-Montería. (12-14 de Octubre de 2018). Feria Empresarial y Tecnologica. Obtenido de https://fetdigital.innovemp.co/\#/

Congreso de Colombia. (2006). LEY 1014 DE 2006. Obtenido de http://www.secretariasenado.gov.co/senado/basedoc/ley_1014_2006.html

CREA Tecnologico de Monterrey. (2011). Centro de recursos para la escritura academica. Obtenido de http://sitios.ruv.itesm.mx/portales/crea/mapa_sitio.htm

Cuevas, J. (5 de octubre de 2018). El proceso del Desing Thinking en el aprendizaje de la competencia "sentido de iniciativa y espirito emprendedor". Obtenido de http://ciriec.es/wp-content/uploads/2018/09/COMUN-140-TD-CUEVAS.pdf

Diccionario de Acción Humanitaria y Cooperación al Desarrollo. (2006). Investigación-acción participativa (IAP). Obtenido de http://www.dicc.hegoa.ehu.es/listar/mostrar/132

DIGITAL BANK. (29 de Mayo de 2018). Obtenido de Transmisión en directo Digital Bank Bogota 2018 día 1: https://www.youtube.com/watch?v=1FJQxC1gOK0

Edinson, H., Smorsgard, N., Wang, X., \& Abrahamsson, P. (2018). Lean Internal Startups for Software Product Innovation in Large Companies: Enablers and Inhibitors. The Journal of Systems and Software.

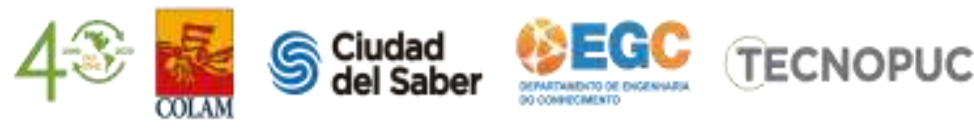


Esteban, E. (2016). Importancia del desarrollo de habilidades académicas como estrategia para el inicio de la formulación y evaluación de proyectos tecnológicos. Obtenido de https://doi.org/10.16925/greylit.1848

Fernández, A. (2006). Metodologías activas para la formación de competencias. Educatio siglo $X X I, 24 \cdot 2006$, pp. 35 - 56.

INDESCO. (2018). Informe Premio Rymel Serrano Uribe 2018. Obtenido de https://www.ucc.edu.co/indesco/Documents/PREMIO-RYMEL-SERRANO-2018indesco.pdf

MinTic. (22 de Junio de 2016). Apps.co. Obtenido de https://apps.co/acerca/appsco/\#8

Paños, J. (2017). Educación emprendedora y metodologías activas para su fomento. Revista Electrónica Interuniversitaria de Formación del Profesorado., 20 (3), 33--48. DOI:http://dx.doi.org/10.6018/reifop.20.3.272221.

Revista emprendedores . (2017). Datos sobre emprendimiento digital en Colombia. Obtenido de

http://revistaemprendedores.co/index.php?option=com_content\&view=article\&id=403 :datos-sobre-emprendimiento-digital-en-colombia\&catid=41:cell-phones-apdas \&Itemid $=109$

Romero, E., Artal, J., García, J., \& Artacho, J. (2017). Aprendizaje invertido con Elevator Pitch y Pecha Kucha. Obtenido de https://zaguan.unizar.es/record/62967/files/139.pdf

Saíz, M., \& Montero, E. (2016). Metodologías activas en docencia universitaria: diseño de una asignatura de ciencias de la salud en la plataforma virtual. Burgos: Universidad de Burgos.

SiKUANI.NET. (2018). Obtenido de https://app.sikuani.net/projects/538

Transformación digital del sector productivo. (2018). Obtenido de https://hackathon.innovemp.co/\#/

Unigarro, M. (2017). Un modelo educativo crítico con enfoque de competencias. Obtenido de https://revistas.ucc.edu.co/index.php/dotr/article/view/1833/1921

UNIMINUTO. (2017). Estrategias y técnicas didácticas. Obtenido de http://www.uniminuto.edu/web/uvd/estrategias-y-tecnicas-didacticas

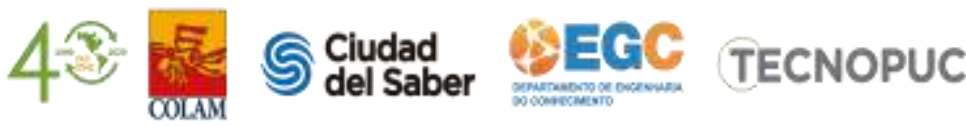



conocimiento e innovación

UniMOOC. (1 de Diciembre de 2014). ¿Que es Lean Startup? Obtenido de https://unimooc.com/que-es-lean-startup/ 